\title{
Potential of epigenetic biomarker O6-methylguanine-DNA methyltransferase gene in glioma
}

\author{
(1) Wei Chiang Goh", (1) Revathy Murali ${ }^{1}$, (1) Shaharum Shamsuddin², (1) Badrisyah Idris ${ }^{1,3}$, (1) Zamzuri Idris ${ }^{1,3}$, \\ (1) Farizan Ahmad ${ }^{1,3}$
}

${ }^{1}$ Department of Neurosciences, School of Medical Sciences, Universiti Sains Malaysia, Kelantan, Malaysia

2School of Health Sciences, Universiti Sains Malaysia, Kelantan, Malaysia

3Hospital Universiti Sains Malaysia, Kelantan, Malaysia

\section{Date submitted:}

24.12.2020

Date accepted:

31.01.2021

Online publication date:

15.12.2021

\section{Corresponding Author:}

Farizan Ahmad, PhD, Department of Neurosciences, School of Medical Sciences, Universiti Sains Malaysia, Kelantan, Malaysia

farizan@usm.my

ORCID:

orcid.org/0000-0001-6413-7530

Keywords: Glioma, O6methylguanine-DNAmethyltransferase, methylation, bisulfide treatment, Malaysia

Presented in: This study was approved by the Human Research Ethics Committee of Universiti Sains Malaysia (JePeM) (ref. no. USM/ JEPeM/17050255).

\begin{abstract}
Aims: Glioblastomas are the most malignant gliomas in adults with the median survival of 15 months only. O6-methylguanine-DNA methyltransferase (MGMT) is a DNA repair enzyme which overcomes the alkylating chemotherapy effect resulting in chemo-resistance. Methylation at the MGMT gene promoter reduces the gene expression and enhances chemosensitivity in cancer treatments. Therefore, this study aimed to screen MGMT methylation status for a potential epigenetic biomarker in glioma detection and treatmentin glioma patients at Hospital Universiti Sains Malaysia.
\end{abstract}

Methods: Forty-one glioma paraffin-embedded glioma tissue samples consisting of grade 2 $(n=11), 3(n=10)$ and $4(n=20)$ were analyzed in this retrospective study. The extracted DNA was subjected to bisulfite treatment and the methylation status was determined via methylationspecific polymerase chain reaction targeting the MGMT gene promoter.

Results: It was observed that $92.7 \%$ of the glioma samples showed methylated and $7.3 \%$ unmethylated MGMT promoter. All grade 2 and grade 3 gliomas showed methylation, compared to $85 \%$ of grade 4 ( $p=0.183$ ). More older glioma patients ( $>40$ years) had methylation compared to younger patients ( $\leq 40$ years) $(95.8 \%$ vs $88.2 \%)(p=0.357)$. More males had methylation compared to females ( $96 \%$ vs $87.5 \%)(p=0.308)$.

Conclusions: MGMT promoter methylation was found predominant in older ( $>40$ years) male patients with grade 2 and 3 gliomas. High percentages of gliomas, $92.7 \%$ harboring methylated MGMT promoter, indicate that it is a potential epigenetic biomarker for glioma detection.

\section{Introduction}

Glioma is a type of brain tumor that begins in glial cells. It is the most predominant type primary brain tumor which can be found in adults, constituting $30 \%$ to $40 \%$ of all intracranial tumors, and its utmost prevalence is between the ages of 40 and 65 years (1). In Malaysia, the annual incidence of central nervous system tumors was predicted at 2.8 per 100,000 people by Globocan 2012 (2). Grade 1 tumors are normally benign and can be treated through complete surgical excision. The median survival is 57 months for grade 2 astrocytoma and 24 months for grade 3 astrocytoma (3). About less than $5 \%$ of the grade 4 
glioblastoma multiforme (GBM) patients were able to live for 5 years after diagnosis (4).

O6-methylguanine methyltransferase (MGMT) gene is located at the chromosome 10q26 encoding 207 amino acids that reverse the alkylation effect at the $\mathrm{O} 6$ position of guanine (5). Alkylating agents induce methylation at guanine that forms mismatch with thymine which subsequently leads to futile mismatch repair cycle, single-strand DNA breakage and cancer cell apoptosis (6). Removal of O6-methylguanine DNA adduct by the MGMT enzyme prevents the mismatch repair cycle and cancer cell death. Reduction of MGMT protein was able to increase chemosensitivity in high-grade gliomas (7). Another similar study reported that xeroderma pigmentosum fibroblasts transfected with DNA repair protein 06-alkylguanineDNA alkyltransferase (ATase) were able to rescue the cells from temozolomide (TMZ)-induced damage and lead to resistance (8). Methylation at the MGMT promoter region spanning $1.2 \mathrm{~kb}$ results in MGMT gene silencing (6).

TMZ is an alkylating agent used to treat newly diagnosed and recurrent GBMs. Even with the current advance treatments, patients with stage 4 glioma, GBM, who completed the treatments, have $90 \%$ of recurrence rate and recurrent GBMs showed resistance towards previous treatments regimes (9). MGMT promoter methylation showed better survival rate in GBM patients with TMZ drug and radiotherapy as compared to radiotherapy only (21.7 vs 15.3 months, $p$ value $=0.007$ ) (10). MGMT methylated GBM patients were associated with better median progression-free survival and overall survival as compared to MGMT unmethylated GBM patients [(8.7 vs 5.7 months, $p$ value <0.0001) and $(21.2$ vs 14 months, $p$ value $<0.0001)$, respectively] (11).

Failure to identify the specific molecular identity of tumor can result in ineffective treatment and may worsen the prognosis (12). Response to the treatments of glioma patients is mostly dependent on the molecular characteristic of the tumors (13). Although MGMT promoter methylation may hold valuable diagnostic and prognostic power in gliomas, the screening test of MGMT promoter in gliomas is yet to be introduced in clinical setting due to lack of MGMT methylation data in gliomas. Therefore, we sought to determine the MGMT promoter methylation status of glioma patients in Hospital Universiti Sains Malaysia, as a potential epigenetic biomarker for diagnostic and treatment stratification to improve the treatment efficacy of current gliomas.

\section{Methods}

\section{Tumor Samples}

Forty-one archived formalin-fixed paraffin-embedded (FFPE) glioma samples consisting of grade $2(n=11)$, grade $3(n=10)$ and grade $4(n=20)$ were collected from Pathology Department,
Hospital Universiti Sains Malaysia. This study is a retrospective study using archived paraffin embedded tissue whereby the protocols received approval by the Human Research Ethics Committee of Universiti Sains Malaysia (JePeM) (ref. no. USM/ JEPeM/17050255). Prior to DNA extraction, a neuropathologist reviewed the glioma samples to confirm the tumor types and gradings based on the 2016 World Health Organization criteria (14).

\section{DNA Extraction}

Two slices of FFPE glioma blocks, with $5 \mu \mathrm{m}$ thickness each, were used to extract genomic DNA using GeneJET FFPE DNA Purification kit (Thermo Scientific, USA) according to the manufacturer's instructions. The DNA concentration and purity were determined using NanoDrop 2000 Spectrophotometer (Thermo Fisher Scientific, USA) and kept at $-20^{\circ} \mathrm{C}$.

\section{Bisulfite DNA Treatment}

Commercially available methylated and unmethylated DNA were used as positive controls in the methylation-specific polymerase chain reaction (PCR) (MSP). CpG Methylated HeLa Genomic DNA (New England Biolab, New England) and 5-Aza-dc Treated Jurkat Genomic DNA (New England Biolab, New England) were used as methylated and unmethylated positive controls, respectively. All extracted DNA and controls were subjected to bisulfite treatment using EpiMark Bisulfite Conversion Kit (New England Biolab, New England) according to the manufacturer's instructions. Approximately $10 \mu \mathrm{L}$ of 100 ng DNA was mixed with $130 \mu \mathrm{L}$ of bisulfite mixture and subjected to PCR. Thermocycling conditions were $95^{\circ} \mathrm{C}$ for 30 seconds, followed by 40 cycles of $95^{\circ} \mathrm{C}$ for 15 seconds, annealing for 30 seconds at $50{ }^{\circ} \mathrm{C}$ and extension at $68^{\circ} \mathrm{C}$ for 1 minute, and finally $68{ }^{\circ} \mathrm{C}$ for 5 minutes. This treatment converted unmethylated cytosine nucleotide $(\mathrm{C})$ to uracil $(\mathrm{U})$ whereas methylated cytosine nucleotide (5-mC) remained unchanged.

\section{Methylation-specific PCR}

All bisulfite-treated DNAs were subjected to MSP using EpiMark HotStart Taq DNA polymerase (New England Biolab, New England) to determine the methylation status at the MGMT promoter. Every treated DNA samples was subjected to two different sets of primers, methylated and unmethylated primers to detect the presence of methylated and unmethylated at the MGMT promoter region (15). Methylated primer sequences, forward: 5'- TTTCGACGTTCGTAGGTTTTCGC-3' and reverse: 5'-GCACTCTTCCGAAAACGAAACG-3' amplifies 81 bp PCR amplicon whereas unmethylated primer sequences, forward: 5'-TTTGTGTTTTGATGTTTGTAGGTTTTTGT-3' and reverse: 5'-AACTCCACACTCTTCCAAAAACAAAACA-3' amplifies 93 bp PCR amplicon respectively.

PCR amplification was performed in a total of $25 \mu \mathrm{L}$ reaction containing $100 \mathrm{ng}$ bisulfite-treated DNA, $2.5 \mu \mathrm{L}$ of 10X EpiMark 
HotStart Taq Reaction Buffer, $2 \mu \mathrm{L}$ of $25 \mathrm{mM} \mathrm{MgCl}, 0.5 \mu \mathrm{L}$ of 10 $\mathrm{mM}$ dNTP each, $0.5 \mu \mathrm{L}$ of $10 \mu \mathrm{M}$ forward and reverse primers and $0.5 \mu \mathrm{L}$ of EpiMark HotStart Taq DNA Polymerase enzyme. Thermocycling conditions were $95^{\circ} \mathrm{C}$ for 5 minutes, followed by 45 cycles of $95^{\circ} \mathrm{C}$ for 30 seconds, annealing for 1 minute at 60 ${ }^{\circ} \mathrm{C}$ and extension at $72{ }^{\circ} \mathrm{C}$ for 30 seconds, and finally $72{ }^{\circ} \mathrm{C}$ for 5 minutes. The PCR products were analyzed using $2 \%$ agarose gel.

Normal brain cells were known to harbor unmethylated MGMT promoter only (6). Gliomas samples are often contaminated with normal tissue due to its infiltrative nature (16). Therefore, glioma samples with both methylated and unmethylated MGMT promoter were considered as methylated MGMT promoter as the unmethylated gene was from the normal brain tissue.

\section{Statistical Analysis}

The statistical analysis was carried out using GraphPad Prism software version 5 (GraphPad Software, USA). The association of MGMT methylation status with the clinicopathological parameters such as tumor grades, age, gender, and race of the patients was determined using the chi-square test. Statistical significance was defined as $p<0.05$.

\section{Results}

The study included 41 glioma samples (age, mean \pm standard deviation: $42.0 \pm 16.8$ male sex: $61.0 \%$ ). All glioma samples were subjected to MSP amplification as shown in Figure 1. Analysis of MGMT gene promoter in 41 glioma samples showed either unmethylated only or a mix of both unmethylated and methylated status. Samples with unmethylated MGMT promoter only showed a single MSP amplicon of $93 \mathrm{bp}$. On the other hand, glioma samples with both methylated and unmethylated MGMT promoter showed $81 \mathrm{bp}$ (methylated) and $93 \mathrm{bp}$ (unmethylated), respectively.

It was observed that $92.7 \%$ of glioma samples showed methylated and $7.3 \%$ unmethylated MGMT promoter. All grade

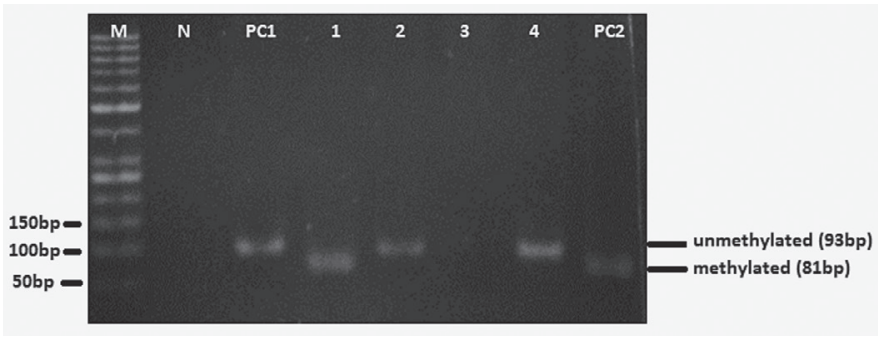

Figure 1. A representative of methylation-specific polymerase chain reaction result of MGMT promoter in gliomas. Lane M: 50 bp DNA ladder, Lane N: Negative control (without DNA), Lane PC1: Positive unmethylated 5-Aza-dc Treated Jurkat Genomic DNA control, Lane 1 and 2: Representative of methylated and unmethylated of a glioma sample, Lane 3 and 4: Representative of unmethylated of a glioma sample, Lane PC2: Positive methylated CpG Methylated HeLa Genomic DNA control
2 and grade 3 gliomas showed methylation, compared to $85 \%$ of grade $4(p=0.183)$. More older glioma patients ( $>40$ years) had methylation compared to younger patients ( $\leq 40$ years) $(95.8 \%$ vs $88.2 \%)(p=0.357)$. More males had methylation compared to females $(96 \%$ vs $87.5 \%) \quad(p=0.308)$. The correlation of MGMT promoter methylation status with the clinicopathological parameters such as tumors grading, age, gender, and the race was shown in Table 1.

\section{Discussion}

According to the Malaysian National Cancer Registry 20072011, brain tumors are the second most common cancer among adults and children respectively (17). The current survival rate of stage 4 GBM is still at dismay level. The median overall survival was 6.5 months and median progression-free survival was 5.5 months only (18). Therefore, improving the current cancer therapeutic methods is important to prolong the survival rate. Cancer biomarkers have been extensively studied as it can predict responsiveness to medical treatments, patient's survival rate and disease diagnosis (19). Thus, new biomarker is needed to improve the current diagnosis and treatment stratification.

It was found that reduced MGMT protein expression via promoter methylation showed a significant improvement in the patient's survival rate and responsiveness to alkylating drug treatment (20). GBM patients with MGMT methylated undergone radiation showed longer median survival compared to the MGMT unmethylated with radiation (15.3 months vs 11.8 months). Together with TMZ and radiation, MGMT methylated GBM patients showed much higher median survival compared to MGMT unmethylated GBM (23.4 months vs 12.6 months) (21). Hence, MGMT promoter methylation status has a high potential for treatment stratification in glioma patients (22-24).

Lack of MGMT promoter methylation data in gliomas and ambiguous testing procedure in hospital has impeded the implementation of this potential biomarker in our local clinical setting. Thus, this study was conducted to determine the current methylation status of MGMT promoter in our local glioma samples. Besides, this study can contribute to the prevalence of MGMT glioma database in Malaysia and globally.

Some glioma samples that showed both methylated and unmethylated MGMT promoter were considered as methylated MGMT due to the infiltrative nature of gliomas. There is no distinct border between the glioma and normal brain tissue, thus the glioma samples are often contaminated with normal brain tissue. This leads to the presence of normal tissue's DNA in the extracted tumor genetic materials (25). Unmethylated MGMT gene in normal tissues produces MGMT protein to repair the damaged DNA (6).

Majority of the glioma samples $(92.7 \%)$ have methylated MGMT promoter. Although there is no significant difference between the tumor grades and MGMT promoter methylation 


\begin{tabular}{|c|c|c|c|c|}
\hline \multirow[b]{2}{*}{ Characteristics } & \multirow{2}{*}{$\begin{array}{l}\text { Total no. of } \\
\text { samples }\end{array}$} & \multicolumn{2}{|c|}{ Methylation status } & \multirow{2}{*}{$p$ value } \\
\hline & & Unmethylated & Methylated & \\
\hline \multicolumn{5}{|l|}{ Tumor grading, $\mathrm{n}(\%)$} \\
\hline Grade 2 & $11(26.8)$ & 0 & $11(100)$ & 0.183 \\
\hline \multicolumn{5}{|l|}{ Age, years } \\
\hline Mean $\pm S D$ & $42.0 \pm 16.8$ & & & \\
\hline$\leq 40, \mathrm{n}(\%)$ & $17(41.5)$ & $2(11.8)$ & $15(88.2)$ & \multirow{2}{*}{0.357} \\
\hline$>40, \mathrm{n}(\%)$ & $24(58.5)$ & $1(4.2)$ & $23(95.8)$ & \\
\hline \multicolumn{5}{|l|}{ Race, n (\%) } \\
\hline Malay & $40(97.6)$ & $3(7.5)$ & $37(92.5)$ & \multirow{2}{*}{0.229} \\
\hline Chinese & $1(2.4)$ & 0 & 0 & \\
\hline
\end{tabular}

status ( $p=0.183$ ), we found that all grade 2 and 3 gliomas had methylated MGMT promoter compared to grade 4 (85\%). On contrary, a study from Europe found that approximately $44.7 \%$ of grade 4 gliomas had MGMT promoter methylation (10). A study from China showed that $58.6 \%$ of GBM showed MGMT promoter methylation (26). Based on the GBM group, most of our local sample had MGMT promoter methylation (85\%) compared to the studies from China (58.6\%) and Europe (44.7\%).

Approximately $88.2 \%$ of younger glioma patients $(\leq 40$ years old) exhibited MGMT promoter methylation compared to unmethylated MGMT (11.8\%). About $95.8 \%$ of older glioma patients (>40 years old) also exhibited MGMT promoter methylation compared to unmethylated MGMT (4.2\%). Most of the young and old glioma patients showed MGMT promoter methylation but slightly more older glioma patients had methylated MGMT promoter compared to younger patients $(95.8 \%$ vs $88.2 \%)$. There was no significant association between the patient's age and MGMT promoter methylation status ( $p=0.357$ ). A study showed that more older GBM patients ( $\geq 50$ years) were found to harbor methylated MGMT compared to the younger GBM patients (61.3\% vs $38.7 \%, p=0.444)(26)$. However, Cancer Genome Atlas project's found that younger patients were associated with glioma CpG island methylation phenotype (G-CIMP) (27).

Our study found that more male patients had methylated MGMT promoter as compared to female patients $(96 \%$ vs $87.5 \%$ ). However, there is no significance between gender and MGMT promoter methylation status $(p=0.308)$. The role of gender in determining glioma prognosis remained ambiguous.
The previous study from Italy showed that female GBM patients with methylated MGMT promoter were found to have better survival rate compared to methylated MGMT males GBM patients $(p=0.028)$ but there was no significance between the unmethylated females and males $(p=0.395)(28)$.

One of the limitations of this study is selection bias. This was because only available FFPE glioma blocks from Pathology Department were selected for this study. This experimental setting was done at the east coast region of Malaysia and the majority race in that population was Malays (29). Thus, this may be explained why most of the samples obtained were from Malays race $(97.6 \%)$ and only one from the Chinese race $(2.4 \%)$. Besides, different genetic makeup between Asian and non-Asian may also contribute to the different distribution of MGMT promoter methylation in gliomas.

Besides, our small sample size may affect the findings as other glioma studies involve larger sample size such as 169 (28), 206 (10) and 573 (20). As the samples were collected at only one location, the sample size can be improved by collecting from different locations and equal distribution of races in Malaysia to produce more accurate information regarding this potential biomarker.

Moreover, sophisticated equipment can be used to improve the quality of the data in this study as well. Pyrosequencing can detect a very small amount of tumor DNA $(5 \%)$ in the background of normal DNA. Since glioma is an infiltrative tumor with no distinct border, it would be best to use sophisticated equipment to capture the true tumor genetic nature. 


\section{Conclusion}

In conclusion, the MGMT promoter methylation was predominant in grade 2 and 3 glioma patients, older age (>40 years) and male gender. Nevertheless, this study showed that a high number of glioma samples from Kelantan, Malaysia harbored MGMT promoter methylation. Therefore, this gene has great potential in diagnosis and treatment stratification to improve glioma patient's survival rate.

\section{Ethics}

Ethics Committee Approval: The study were approved by the Human Research Ethics Committee of Universiti Sains Malaysia (JePeM) (ref. no. USM/JEPeM/17050255).

Informed Consent: Retrospective study.

Peer-review: Externally peer-reviewed.

\section{Authorship Contributions}

Concept: F.A., Design: Z.I., F.A., Data Collection or Processing: R.M., Analysis or Interpretation: S.S., B.I., Literature Search: W.C.G., Writing: W.C.G., B.I., Z.I.

Conflict of Interest: No conflict of interest was declared by the authors.

Financial Disclosure: This work was supported by the USM Short-Term grant (304/PPSP/6315115) and the USM Fellowship provided by Universiti Sains Malaysia.

\section{References}

1. Schneider T, Mawrin C, Scherlach C, Skalej M, Firsching R. Gliomas in adults. Dtsch Arztebl Int. 2010;107:799-807.

2. Brain and spinal tumour. Med J Malaysia. 2014;69:261267.

3. Dong X, Noorbakhsh A, Hirshman BR, et al. Survival trends of grade I, II, and III astrocytoma patients and associated clinical practice patterns between 1999 and 2010: A SEERbased analysis. Neurooncol Pract. 2016;3:29-38.

4. Aum DJ, Kim DH, Beaumont TL, Leuthardt EC, Dunn GP, $\mathrm{Kim} \mathrm{AH}$. Molecular and cellular heterogeneity: the hallmark of glioblastoma. Neurosurg Focus. 2014;37:E11.

5. Pegg AE, Dolan ME, Moschel RC. Structure, function, and inhibition of O6-alkylguanine-DNA alkyltransferase. Prog Nucleic Acid Res Mol Biol. 1995;51:167-223.

6. Sharma S, Salehi F, Scheithauer BW, Rotondo F, Syro LV, Kovacs K. Role of MGMT in tumor development, progression, diagnosis, treatment and prognosis. Anticancer Res. 2009;29:3759-3768.

7. Sarkaria JN, Kitange GJ, James CD, et al. Mechanisms of chemoresistance to alkylating agents in malignant glioma. Clin Cancer Res. 2008;14:2900-2908.

8. Baer JC, Freeman AA, Newlands ES, Watson AJ, Rafferty JA, Margison GP. Depletion of O6-alkylguanineDNA alkyltransferase correlates with potentiation of temozolomide and CCNU toxicity in human tumour cells. Br J Cancer. 1993;67:1299-1302.

9. Lau D, Magill ST, Aghi MK. Molecularly targeted therapies for recurrent glioblastoma: current and future targets. Neurosurg Focus. 2014;37:E15.

10. Hegi ME, Diserens AC, Gorlia T, et al. MGMT gene silencing and benefit from temozolomide in glioblastoma. $\mathrm{N}$ Engl $\mathrm{J}$ Med. 2005;352:997-1003.

11. Gilbert MR, Wang M, Aldape KD, et al. Dose-dense temozolomide for newly diagnosed glioblastoma: a randomized phase III clinical trial. J Clin Oncol. 2013;31:4085-4091.

12. Ray S, Bonafede MM, Mohile NA. Treatment Patterns, Survival, and Healthcare Costs of Patients with Malignant Gliomas in a Large US Commercially Insured Population. Am Health Drug Benefits. 2014;7:140-149.

13. Wang J, Zhao YY, Li JF, et al. IDH1 mutation detection by droplet digital PCR in glioma. Oncotarget. 2015;6:3965139660 .

14. Louis DN, Perry A, Reifenberger G, et al. The 2016 World Health Organization Classification of Tumors of the Central Nervous System: a summary. Acta Neuropathol. 2016;131:803-820.

15. Yoshioka M, Matsutani T, Hara A, et al. Real-time methylation-specific PCR for the evaluation of methylation status of MGMT gene in glioblastoma. Oncotarget. 2018;9:27728-27735.

16. Boisselier $B$, Marie $Y$, Labussière $M$, et al. COLD PCR HRM: a highly sensitive detection method for IDH1 mutations. Hum Mutat. 2010;31:1360-1365.

17. Summary of Malaysian National Cancer Registry Report 2007-2011. Vol. 1. 2015. Available from: https://www. crc.gov.my/wp-content/uploads/documents/report/ MNCRRrepor2007-2011.pdf

18. van Linde ME, Brahm CG, de Witt Hamer PC, et al. Treatment outcome of patients with recurrent glioblastoma multiforme: a retrospective multicenter analysis. J Neurooncol. 2017;135:183-192.

19. Henrya NL, Hayes Daniel F. Cancer biomarkers. Cancer Biomarkers. 2011;6:1-273.

20. Stupp R, Mason WP, van den Bent MJ, et al. Radiotherapy plus concomitant and adjuvant temozolomide for glioblastoma. N Engl J Med. 2005;352:987-996.

21. Stupp R, Hegi ME, Mason WP, et al. Effects of radiotherapy with concomitant and adjuvant temozolomide versus radiotherapy alone on survival in glioblastoma in a randomised phase III study: 5-year analysis of the EORTCNCIC trial. Lancet Oncol. 2009;10:459-466.

22. Brandes AA, Franceschi E, Paccapelo A, et al. Role of MGMT Methylation Status at Time of Diagnosis and Recurrence for Patients with Glioblastoma: Clinical Implications. Oncologist. 2017;22:432-437. 
23. Esteller M, Corn PG, Baylin SB, Herman JG. A gene hypermethylation profile of human cancer. Cancer Res. 2001;61:3225-3229.

24. Ting AH, Jair KW, Schuebel KE, Baylin SB. Differential requirement for DNA methyltransferase 1 in maintaining human cancer cell gene promoter hypermethylation. Cancer Res. 2006;66:729-735.

25. Goh WC, Idris B, Kandasamy R, Shamsuddin S, Jaafar H, Ahmad F. PCR-RFLP method enhance DNA sequencing of IDH1 somatic mutations detection in gliomas. Gulhane Med J. 2019;61:167-171.

26. Shen $D$, Liu $T$, Lin $Q$, et al. MGMT promoter methylation correlates with an overall survival benefit in Chinese highgrade glioblastoma patients treated with radiotherapy and alkylating agent-based chemotherapy: a single-institution study. PLoS One. 2014;9:e107558.

27. Verhaak RG, Hoadley KA, Purdom E, et al. Integrated genomic analysis identifies clinically relevant subtypes of glioblastoma characterized by abnormalities in PDGFRA, IDH1, EGFR, and NF1. Cancer Cell. 2010;17:98-110.

28. Franceschi E, Tosoni A, Minichillo S, et al. The Prognostic Roles of Gender and O6-Methylguanine-DNA Methyltransferase Methylation Status in Glioblastoma Patients: The Female Power. World Neurosurg. 2018;112:e342-347.

29. Dzali NBM, Zahary MN, Bakar NH, et al. Distribution pattern of brain tumour in a tertiary hospital in east coast, Malaysia. Malaysian J Public Heal Med. 2017;2:41-48. 